\title{
ON THE PERFORMANCE OF SEMIDEFINITE RELAXATION MIMO DETECTORS FOR QAM CONSTELLATIONS
}

\author{
Anthony Man-Cho So* \\ Department of Systems Engineering and Engineering Management \\ The Chinese University of Hong Kong \\ Shatin, N. T., Hong Kong
}

\begin{abstract}
Due to their computational efficiency and strong empirical performance, semidefinite relaxation (SDR)-based algorithms have gained much attention in multiple-input multiple-output (MIMO) detection. In the case of a binary phase-shift keying (BPSK) constellation, the theoretical performance of the SDR approach is relatively well-understood. However, little is known about the case of quadrature amplitude modulation (QAM) constellations, although simulation results suggest that the SDR approach should work well in the low signal-to-noise ratio (SNR) region. In this paper we make a first step towards explaining such phenomenon by showing that in the case of QAM constellations, several commonly used SDR-based algorithms will provide a constant factor approximation to the optimal log-likelihood value in the low SNR region with exponentially high probability. Our result gives some theoretical justification for using SDR-based algorithms for the MIMO detection of QAM signals, at least in the low SNR region.
\end{abstract}

Index Terms- Multiple-Input Multiple-Output (MIMO) Detection, Quadrature Amplitude Modulation (QAM), Semidefinite Relaxation, Performance Analysis, Approximation Algorithm

\section{INTRODUCTION}

As is well-known, multiple antennae communication systems can provide substantial performance gain over their single antenna counterparts (see, e.g., [13]). In order to fully realize such gain, however, the receiver must be able to detect the vector of transmitted symbols in an efficient manner. Consequently, the problem of multiple-input multiple-output (MIMO) detection has received considerable attention over the years. For a linear channel that satisfies certain standard assumptions, a symbol vector that minimizes the error probability can be found by solving the maximum-likelihood (ML) detection problem (see, e.g., [16]). Unfortunately, the ML detection problem is NP-hard in the worst case [15], and thus an efficient (i.e. polynomial-time) algorithm for solving it is not likely to exist. In fact, currently there is not even an efficient algorithm for solving the ML detection problem in the MIMO setting, where the channel is assumed to follow certain stochastic model (and hence is not completely arbitrary). As a result, many sub-optimal but efficient heuristics have been proposed for solving the ML detection problem (see, e.g., [1] for a brief overview). One such heuristic called the semidefinite relaxation (SDR) detector - has attracted a lot of interest recently (see, e.g., $[12,5,17,1,10,8,7,18,6]$ ). Roughly speaking, the SDR detector solves a convex relaxation of

${ }^{*}$ E-mail: manchoso@se. cuhk. edu.hk. The author would like to thank Professor Ken Ma for many insightful discussions. the ML detection problem, which can be expressed as a semidefinite program (SDP). As such, the SDR detector is computationally efficient. Moreover, in the case of a binary phase-shift keying (BPSK) constellation, the theoretical performance of the SDR detector is fairly well-understood. For instance, it is known that the SDR detector will provide a constant factor approximation to the optimal $\log$-likelihood value in the low signal-to-noise ratio (SNR) region almost surely [3], and that it achieves full receive diversity [2]. Unfortunately, the analyses presented in $[3,2]$ depend crucially on the structure of the channel matrix. As a result, they do not extend to cover the case of quadrature amplitude modulation (QAM) constellations. In fact, little is known about the theoretical performance of the SDR detector in the case of QAM constellations, although simulation results suggest that it should work well in the low SNR region.

Our goal in this paper is to make a first step towards remedying such a situation. Specifically, we introduce a framework for analyzing the performance of the SDR detector for QAM constellations. We show that for the $4^{q}$-QAM constellation, where $q \geq 1$ is fixed, a version of the SDR detector will provide a constant factor approximation to the optimal log-likelihood value in the low SNR region with high probability. In fact, we can show that the probability approaches 1 exponentially fast as the channel size increases. Together with a recent result of Ma et al. [6], we see that in the case of QAM constellations, the same is true for several other commonly used SDR detectors. Our work is similar in spirit to that of Kisialiou and Luo [3]. However, they differ in that our analysis is non-asymptotic in nature, which allows us to determine the performance of the SDR detector when the channel size is finite. Moreover, our techniques are more general in the sense that they do not have a strong dependence on the structure of the channel matrix. As a result, we are able to analyze the performance of the SDR detector not only for the QAM constellations but also for some other signal constellations (see, e.g, [11]).

\section{PRELIMINARIES}

\subsection{The MIMO Detection Problem}

Consider an MIMO system with $\tilde{n}$ transmit and $\tilde{m}$ receive antennae (with $\tilde{m} \geq \tilde{n}$ ), whose input-output relationship is modeled as:

$$
\tilde{y}=\sqrt{\rho / \tilde{n}} \tilde{H} \tilde{x}+\tilde{v}
$$

Here, $\tilde{H} \in \mathbb{C}^{\tilde{m} \times \tilde{n}}$ is the channel matrix whose entries are independent standard complex Gaussian random variables (see [13, Appendix A]); $\tilde{v} \in \mathbb{C}^{\tilde{m}}$ is an additive white Gaussian noise with unit variance, i.e. $\tilde{v}$ is a standard circular symmetric complex Gaussian random vector that is independent of $\tilde{H} ; \rho>0$ is the (appropriately 
scaled) SNR per receive antenna; $\tilde{y} \in \mathbb{C}^{\tilde{m}}$ is the vector of received signals; and $\tilde{x} \in \mathcal{S}^{\tilde{n}}$ is the vector of transmitted symbols whose entries are chosen from some constellation set $\mathcal{S} \subset \mathbb{C}$. In this paper we shall focus on the case where $\mathcal{S}$ is the $4^{q}-\mathrm{QAM}$ constellation set for some fixed integer $q \geq 1$, i.e. $\mathcal{S}=\left\{s_{R}+j s_{I}: s_{R}, s_{I} \in\right.$ $\left.\left\{ \pm 1, \pm 3, \pm 5, \ldots, \pm\left(2^{q}-1\right)\right\}\right\}$. It would be convenient for our subsequent exposition to reformulate the complex-valued model (1) into an equivalent real-valued model. Towards that end, let $n=2 \tilde{n}$ and $m=2 \tilde{m}$. Define:

$y=\left[\begin{array}{c}\Re(\tilde{y}) \\ \Im(\tilde{y})\end{array}\right], \bar{H}=\sqrt{2}\left[\begin{array}{cc}\Re(\tilde{H}) & -\Im(\tilde{H}) \\ \Im(\tilde{H}) & \Re(\tilde{H})\end{array}\right], v=\left[\begin{array}{c}\Re(\tilde{v}) \\ \Im(\tilde{v})\end{array}\right]$

Note that $\bar{H} \in \mathbb{R}^{m \times n}$ and $y, v \in \mathbb{R}^{m}$, and that the entries of $\bar{H}$ are standard Gaussian random variables. Then, it is straightforward to verify that (1) is equivalent to the following real-valued model:

$$
y=\sqrt{\rho / n} \bar{H} \bar{x}+v
$$

where $\bar{x} \in \mathcal{S}_{q} \equiv\left\{ \pm 1, \pm 3, \pm 5, \ldots, \pm\left(2^{q}-1\right)\right\}^{n}$. Under certain standard assumptions, the ML detection problem associated with (3) is given by:

$$
\min _{\bar{x} \in \mathcal{S}_{q}}\|y-\sqrt{\rho / n} \bar{H} \bar{x}\|_{2}^{2}
$$

An optimal solution to (4) is also known as an optimal ML solution, which has the property that it minimizes the probability of error in the joint detection of the transmitted symbols (see, e.g., [16]). However, it is still not known whether there exists a provably efficient algorithm for computing such a solution.

\subsection{Semidefinite Relaxation of the ML Detection Problem}

In the absence of a provably efficient algorithm, a popular approach for tackling the ML detection problem (4) is to consider its semidefinite relaxation. There are many ways to relax problem (4) into an SDP; see, e.g., [17, 10, 7, 8, 18]. For the sake of simplicity, we shall follow the approach of Mao et al. [7]. We remark that this does not limit the applicability of our results, as the recent work of Ma et al. [6] allows us to transfer those results to other semidefinite relaxations as well.

To begin, observe that for any integer $q \geq 1$, we have:

$$
\mathcal{S}_{q}=\left\{x_{1}+2 x_{2}+\cdots+2^{q-1} x_{q}: x_{1}, \ldots, x_{q} \in\{-1,1\}^{n}\right\}
$$

In other words, given a symbol $s \in\left\{ \pm 1, \pm 3, \ldots, \pm\left(2^{q}-1\right)\right\}$, we can express it as $s=s_{1}+2 s_{2}+\cdots+2^{q-1} s_{q}$, where $s_{1}, \ldots, s_{q} \in$ $\{-1,1\}$. Note that the bits $s_{1}, \ldots, s_{q}$ need not correspond to the actual information bits that are mapped into the symbol $s$. In particular, the following ML detection problem:

$$
v_{m l}=\min _{x \in\{-1,1\}^{q n}}\|y-\sqrt{\rho / n} H x\|_{2}^{2}
$$

where $H=\left[\begin{array}{lllll}\bar{H} & 2 \bar{H} & 4 \bar{H} & \cdots & 2^{q-1} \bar{H}\end{array}\right] \in \mathbb{R}^{m \times q n}-$ which is equivalent to problem (4) due to the representation (5) does not depend on how the information bits are mapped to the symbols. Now, upon homogenization, we may relax problem (6) into the following SDP (see, e.g., [3]):

$$
v_{s d p}=\inf \{\operatorname{tr}(Q X): \operatorname{diag}(X)=\mathbf{1}, X \succeq \mathbf{0}\}
$$

where $\mathbf{1} \in \mathbb{R}^{q n+1}$ is the vector of all ones, and

$$
Q=\left[\begin{array}{cc}
(\rho / n) H^{T} H & -\sqrt{\rho / n} H^{T} y \\
-\sqrt{\rho / n} y^{T} H & \|y\|_{2}^{2}
\end{array}\right] \in \mathbb{R}^{(q n+1) \times(q n+1)}
$$

Since $Q \succeq \mathbf{0}$ and problem (7) is a relaxation of problem (6), we clearly have $0 \leq v_{s d p} \leq v_{m l}$. We should emphasize that both $v_{m l}$ and $v_{s d p}$ depend on the particular realizations of $\bar{H}$ and $v$, since $y$ is related to $\bar{H}$ and $v$ via (3).

Note that the SDP (7) can be solved to any desired accuracy in polynomial time [14], and efficient implementations are available (see, e.g., [4]). However, we still need a rounding procedure that, given any feasible solution $\hat{X} \in \mathbb{R}^{(q n+1) \times(q n+1)}$ to (7), converts it into a feasible solution $\hat{x} \in\{-1,1\}^{q n}$ to (6). Below is one such procedure (cf. [3]):

\section{Randomized Rounding Procedure}

1. Partition the matrix $\hat{X} \in \mathbb{R}^{(q n+1) \times(q n+1)}$ as:

$$
\hat{X}=\left[\begin{array}{cc}
U & u \\
u^{T} & 1
\end{array}\right]
$$

where $u \in \mathbb{R}^{q n}$ and $U \in \mathbb{R}^{q n \times q n}$. Note that since $\hat{X} \succeq \mathbf{0}$ and $\operatorname{diag}(\hat{X})=\mathbf{1}$, we must have $\left|u_{i}\right| \leq 1$ for $i=1, \ldots, q n$.

2. Let $x^{1}, \ldots, x^{m}$ be $m$ independent $q n$-dimensional random vectors, each of whose entries are independent and identically distributed according to the following distribution:

$$
\operatorname{Pr}\left(x_{j}^{i}=1\right)=\frac{1+u_{j}}{2}, \quad \operatorname{Pr}\left(x_{j}^{i}=-1\right)=\frac{1-u_{j}}{2}
$$

where $1 \leq i \leq m$ and $1 \leq j \leq q n$. Set $\hat{x}^{i}=\left(x^{i}, 1\right) \in$ $\mathbb{R}^{q n+1}$, where $i=1, \ldots, m$.

3. Let $i^{*}=\arg \min _{1 \leq i \leq m}\left(\hat{x}^{i}\right)^{T} Q \hat{x}^{i}$, and define $\hat{x}^{\prime}=\hat{x}^{i^{*}}$. Set $v_{s d r}=\hat{x}^{\prime T} Q \hat{x}^{\prime}$, and return $\hat{x}=\left(\hat{x}_{1}^{\prime}, \ldots, \hat{x}_{q n}^{\prime}\right) \in$ $\{-1,1\}^{q n}$ as our candidate solution. Note that $\hat{x}$ is feasible for (6), and that $v_{s d r}=\|y-\sqrt{\rho / n} H \hat{x}\|_{2}^{2}$. It follows that $v_{m l} \leq v_{s d r}$.

Naturally, we are interested in the performance of the above rounding procedure, and one measure is the so-called approximation ratio. Specifically, we would like to establish a probabilistic upper bound on the ratio $v_{s d r} / v_{m l}$, where the probability is computed over all possible realizations of $\bar{H}$ and $v$, as well as the random vectors generated according to (9). We remark that such a performance measure has been used before to evaluate the performance of the SDR detector in the case of an BPSK constellation [3]. Intuitively, if the ratio is close to 1 , then we may conclude that the solution generated by the rounding procedure is close (in terms of the log-likelihood value) to the optimal ML solution. In the next section we show that the aforementioned SDP-based procedure will actually achieve a constant approximation ratio (i.e. independent of $\tilde{m}$ and $\tilde{n}$ ) in the low SNR region with high probability. This gives a strong indication that the SDR detector is a good heuristic for solving the ML detection problem, at least in the low SNR region.

\section{ANALYSIS OF THE SEMIDEFINITE RELAXATION}

To begin, let $q \geq 1$ be fixed, and consider a particular realization of $(\bar{H}, v)$ (and hence of $Q$ ). Let $\hat{X} \in \mathbb{R}^{(q n+1) \times(q n+1)}$ be a feasible solution to (7) with objective value $v_{s d p}$ and partition $\hat{X}$ according to $(8)$. Let $\hat{x}^{1}, \ldots, \hat{x}^{m}$ be the random vectors generated in Step 2 of the randomized rounding procedure. Set $\Gamma \equiv \mathbb{E}_{\hat{x}}\left[\left(\hat{x}^{1}\right)^{T} Q \hat{x}^{1}\right]$, where $\mathbb{E}_{\hat{x}}$ denotes the mathematical expectation w.r.t. the distribution defined in (9). Then, by Markov's inequality and the fact that the random vectors $\hat{x}^{1}, \ldots, \hat{x}^{m}$ are i.i.d., we have:

$$
\operatorname{Pr}_{\hat{x}}\left(v_{s d r} \geq 2 \Gamma\right)=\left[\operatorname{Pr}_{\hat{x}}\left(\left(\hat{x}^{1}\right)^{T} Q \hat{x}^{1} \geq 2 \Gamma\right)\right]^{m} \leq 2^{-m}
$$


Now, let $\hat{u}=(u, 1) \in \mathbb{R}^{q n+1}$, where $u \in \mathbb{R}^{q n}$ is given by (8). Note that:

$$
\Gamma=\mathbb{E}_{\hat{x}}\left[\sum_{i=1}^{q n+1} \sum_{j=1}^{q n+1} Q_{i j} \hat{x}_{i}^{1} \hat{x}_{j}^{1}\right] \leq \hat{u}^{T} Q \hat{u}+\sum_{i=1}^{q n} Q_{i i}
$$

Since $\hat{X} \succeq \mathbf{0}$, we have $U \succeq u u^{T}$ by the Schur complement. It follows that $\hat{X} \succeq \hat{u} \hat{u}^{T}$, whence $\hat{u}^{T} Q \hat{u}=\operatorname{tr}\left(Q \hat{u} \hat{u}^{T}\right) \leq \operatorname{tr}(Q \hat{X})=$ $v_{s d p}$. In particular, we conclude that:

$$
\Gamma \leq v_{s d p}+\frac{\rho}{n} \operatorname{tr}\left(H^{T} H\right) \leq v_{s d p}+\rho q \lambda_{\max }\left(H^{T} H\right)
$$

where $\lambda_{\max }\left(H^{T} H\right)$ is the largest eigenvalue of $H^{T} H$.

Now, if we could show that the values $v_{s d p}$ and $\rho q \lambda_{\max }\left(H^{T} H\right)$ are within a constant factor of each other with high probability (w.r.t. the realizations of $(\bar{H}, v)$ ), then (10) and (11) would imply that $v_{s d r}$ and $v_{s d p}$ (and hence $v_{s d r}$ and $v_{m l}$ ) are within a constant factor of each other with high probability (w.r.t. the realizations of $(\bar{H}, v)$ and $\hat{x})$. To carry out this idea, we first need an estimate on the largest singular value of the random matrix $H$. In fact, as the following proposition shows, it suffices to estimate the largest singular value of $\bar{H}$ :

Proposition 1 Let $H=\left[\begin{array}{lllll}\bar{H} & 2 \bar{H} & 4 \bar{H} & \cdots & 2^{q-1} \bar{H}\end{array}\right] \in$ $\mathbb{R}^{m \times q n}$, where $\bar{H}$ is given by (2). Then, we have $\|H\|_{2}=$ $\sqrt{\left(4^{q}-1\right) / 3} \cdot\|\bar{H}\|_{2}$, where $\|A\|_{2}$ denotes the largest singular value of the matrix $A$.

The proof of Proposition 1 is deferred to the full version of this paper. Now, we use an $\epsilon$-net argument to estimate $\|\bar{H}\|_{2}$ (see, e.g., [9]). We begin with a definition.

Definition 1 Let $D \subset \mathbb{R}^{n}$ and $\epsilon>0$ be fixed. We say that a subset $N \subset D$ is an $\epsilon-$ net of $D$ if for every $p \in D$, there exists an $p^{\prime} \in N$ such that $\left\|p-p^{\prime}\right\|_{2} \leq \epsilon$.

Let $\mathbb{S}^{n-1}=\left\{x \in \mathbb{R}^{n}:\|x\|_{2}=1\right\}$. The following result shows that the largest singular value of an $m \times n$ matrix $A$ can be estimated using appropriate $\epsilon$-nets:

Fact 1 ([9, Proposition 2.3]) Let $\epsilon, \delta>0$ be fixed. Let $N$ be an $\epsilon$-net of $\mathbb{S}^{n-1}$, and let $M$ be an $\delta$-net of $\mathbb{S}^{m-1}$. Then, for any $m \times n$ matrix $A$, we have $\|A\|_{2} \leq(1-\epsilon)^{-1}(1-$ $\delta)^{-1} \sup _{w \in M, z \in N}\left|w^{T} A z\right|$.

Armed with Fact 1, we are ready to establish the following result:

Proposition 2 Let $\bar{H}$ be as in (2). Then, we have:

$$
\underset{\bar{H}}{\operatorname{Pr}}\left(\|\bar{H}\|_{2}>\sqrt{8 m}\right) \leq \exp (-m / 16) \quad \text { for } m \geq 1
$$

Proof (cf. [9, Proposition 2.4]) Let $N$ be an (1/2)-net of $\mathbb{S}^{n-1}$, and let $M$ be an (1/2)-net of $\mathbb{S}^{m-1}$. It is known that these nets can be chosen with $|M| \leq 6^{m}$ and $|N| \leq 6^{n}$. Now, observe that for $w \in M$ and $z \in N$, the random variable $w^{T} \bar{H} z$ is Gaussian with mean 0 and variance 1 . In particular, for any $\theta>0$ and $m \geq 1$, we have:

$$
\underset{\bar{H}}{\operatorname{Pr}}\left(\left|w^{T} \bar{H} z\right|>\theta \sqrt{m}\right) \leq \sqrt{\frac{2}{\pi}} \cdot \frac{1}{\theta \sqrt{m}} \cdot \exp \left(-\frac{\theta^{2} m}{2}\right)
$$

It then follows from Fact 1 and the union bound that:

$$
\underset{\bar{H}}{\operatorname{Pr}}\left(\|\bar{H}\|_{2}>\theta \sqrt{m}\right) \leq \frac{4}{\theta}\left(36 \exp \left(-\frac{\theta^{2}}{2}\right)\right)^{m}
$$

(recall that $m \geq n$ ). The desired result now follows by setting $\theta=$ $\sqrt{8}$.

As a corollary to Propositions 1 and 2, we have:

$$
\underset{\bar{H}}{\operatorname{Pr}}\left(\lambda_{\max }\left(H^{T} H\right)>\frac{8\left(4^{q}-1\right)}{3} m\right) \leq \exp (-m / 16)
$$

Next, we need to show that $v_{s d p}$ is large (on the order of $m$ ) with high probability (w.r.t. the realizations of $(\bar{H}, v)$ ), so that it is comparable to $\lambda_{\max }\left(H^{T} H\right)$. By the SDP weak duality theorem, it suffices to consider the dual of (7) and exhibit a dual feasible solution with large objective value. We remark that such an idea has been used in the work of Kisialiou and Luo [3]. However, our approach differs from that of [3] in that we are able to obtain a non-asymptotic result.

To begin, let us write down the dual of (7):

$$
\sup \left\{\operatorname{tr}(Z): Q-Z \succeq \mathbf{0}, Z \in \mathbb{R}^{(q n+1) \times(q n+1)} \text { diagonal }\right\}
$$

Let $\alpha>0$ and $\beta \in \mathbb{R}$ be parameters to be chosen, and define:

$$
\bar{Z}=\left[\begin{array}{cc}
-\alpha I & \mathbf{0} \\
\mathbf{0}^{T} & \beta
\end{array}\right]
$$

In order for $\bar{Z}$ to be feasible for (14), we must have $Q-\bar{Z} \succeq \mathbf{0}$. By the Schur complement, this is equivalent to:

$$
y^{T}\left[I-\frac{\rho}{n} H\left(\frac{\rho}{n} H^{T} H+\alpha I\right)^{-1} H^{T}\right] y \geq \beta
$$

(note that $(\rho / n) H^{T} H+\alpha I$ is invertible for any $\alpha>0$ ). Now, observe that:

$$
\begin{aligned}
& y^{T}\left[I-\frac{\rho}{n} H\left(\frac{\rho}{n} H^{T} H+\alpha I\right)^{-1} H^{T}\right] y \\
\geq & {\left[1-\frac{\rho}{n} \lambda_{\max }\left(H\left(\frac{\rho}{n} H^{T} H+\alpha I\right)^{-1} H^{T}\right)\right] \cdot\|y\|_{2}^{2} }
\end{aligned}
$$

We bound:

$$
\begin{aligned}
& \lambda_{\max }\left[H\left(\frac{\rho}{n} H^{T} H+\alpha I\right)^{-1} H^{T}\right] \\
\leq & \lambda_{\max }\left(H H^{T}\right) \cdot \lambda_{\max }\left[\left(\frac{\rho}{n} H^{T} H+\alpha I\right)^{-1}\right] \\
\leq & \alpha^{-1} \lambda_{\max }\left(H^{T} H\right)
\end{aligned}
$$

On the other hand, since $\bar{H}$ and $v$ are independent, we see from (3) that each entry of $y$ is a Gaussian random variable with mean 0 and variance $(\rho / n) \sum_{i=1}^{n} \bar{x}_{i}^{2}+(1 / 2)$, and that the entries are independent. Thus, by standard concentration results on the norm of a Gaussian random vector, we have:

$$
\underset{(\bar{H}, v)}{\operatorname{Pr}}\left[\|y\|_{2}^{2}<\frac{1}{2}\left(\rho+\frac{1}{2}\right) m\right] \leq \exp (-m / 16)
$$

Upon putting together (13), (16) and (17), we see that by choosing $\beta=\beta_{0}$, where:

$$
\beta_{0} \equiv \frac{1}{2}\left(1-\frac{8\left(4^{q}-1\right) \rho \gamma}{3 \alpha}\right)\left(\rho+\frac{1}{2}\right) m, \gamma \equiv \frac{m}{n}=\frac{\tilde{m}}{\tilde{n}} \geq 1
$$

the inequality (15) will hold with probability (over all possible realizations of $(\bar{H}, v))$ at least $1-2 \exp (-m / 16)$. Moreover, we have $\operatorname{tr}(\bar{Z})=\beta_{0}-q n \alpha$. Upon optimizing over $\alpha>0$ and noting that $v_{s d p} \geq \operatorname{tr}(\bar{Z})$, we obtain the following result: 
Proposition 3 Let $q \geq 1$ be a fixed integer, and let $\gamma \equiv m / n=$ $\tilde{m} / \tilde{n} \geq 1$. Define:

$$
\alpha=2 \gamma \sqrt{\frac{4^{q}-1}{3 q}\left(\rho+\frac{1}{2}\right)}, \quad \rho_{0}=\frac{3}{2\left(64 q\left(4^{q}-1\right)-3\right)}
$$

and let $\beta_{0}$ be as in (18). Suppose that the SNR $\rho$ satisfies $\rho \in\left(0, \rho_{0}\right)$. Then, with probability at least $1-2 \exp (-m / 16)$, we have:

$$
v_{s d p} \geq\left[\frac{1}{2}\left(\rho+\frac{1}{2}\right)-\sqrt{\frac{8 \rho q(2 \rho+1)\left(4^{q}-1\right)}{3}}\right] m>0
$$

We are now ready to prove the main result of this paper:

Theorem 1 Let $q \geq 1$ be a fixed integer. Suppose that the SNR $\rho$ satisfies $\rho \in\left(0, \rho_{0}\right)$, where $\rho_{0}$ is given by (19). Define:

$\mu_{1}=\frac{8 \rho q\left(4^{q}-1\right)}{3}, \mu_{2}=\frac{1}{2}\left(\rho+\frac{1}{2}\right)-\sqrt{\frac{8 \rho q(2 \rho+1)\left(4^{q}-1\right)}{3}}$

Then, we have:

$$
\underset{(\bar{H}, v, \hat{x})}{\operatorname{Pr}}\left[v_{s d r} \leq 2\left(1+\frac{\mu_{1}}{\mu_{2}}\right) v_{m l}\right] \geq 1-3 \exp (-m / 16)
$$

In particular, in the low SNR region, the SDR detector will produce a constant factor approximate solution to the ML detection problem with exponentially high probability.

Proof By (11), (13), Proposition 3 and the fact that $v_{s d p} \leq v_{m l}$, we have:

$$
\Gamma \leq v_{s d p}+\frac{8 \rho q\left(4^{q}-1\right)}{3} m \leq\left(1+\frac{\mu_{1}}{\mu_{2}}\right) v_{m l}
$$

with probability at least $1-2 \exp (-m / 16)$. The desired result then follows from (10).

Note that Theorem 1, as it is stated, applies only to the particular semidefinite relaxation (originally proposed by Mao et al. [7]) described in Section 2.2. However, using the equivalence result of Ma et al. [6], one can show, among other things, that Theorem 1 actually applies to the semidefinite relaxation developed by Sidiropoulos and Luo [10] as well.

Finally, we should point out that we have made no attempt in optimizing the constants in our proofs. With a more refined analysis, those constants can certainly be improved.

\section{CONCLUSION}

In this paper we gave some theoretical justification for using SDRbased algorithms for the MIMO detection of QAM signals in the low SNR region.

\section{REFERENCES}

[1] J. Jaldén, "Detection for Multiple Input Multiple Output Channels: Analysis of Sphere Decoding and Semidefinite Relaxation," Ph.D. dissertation, School of Electrical Engineering, Royal Institute of Technology (KTH), Stockholm, Sweden, 2006.

[2] J. Jaldén and B. Ottersten, "The Diversity Order of the Semidefinite Relaxation Detector," IEEE Transactions on Information Theory, vol. 54, no. 4, pp. 1406-1422, 2008.
[3] M. Kisialiou and Z.-Q. Luo, "Performance Analysis of QuasiMaximum-Likelihood Detector Based on Semidefinite Programming," in Proceedings of the IEEE International Conference on Acoustics, Speech, and Signal Processing, 2005 (ICASSP 2005), vol. 3, 2005, pp. III-433-III-436.

[4] —, "Efficient Implementation of a Quasi-MaximumLikelihood Detector Based on Semi-Definite Relaxation," in Proceedings of the IEEE International Conference on Acoustics, Speech, and Signal Processing, 2007 (ICASSP 2007), vol. 4, 2007, pp. IV-1329-IV-1332.

[5] W.-K. Ma, T. N. Davidson, K. M. Wong, Z.-Q. Luo, and P.-C. Ching, "Quasi-Maximum-Likelihood Multiuser Detection Using Semi-Definite Relaxation with Application to Synchronous CDMA," IEEE Transactions on Signal Processing, vol. 50, no. 4, pp. 912-922, 2002.

[6] W.-K. Ma, C.-C. Su, J. Jaldén, T.-H. Chang, and C.-Y. Chi, "The Equivalence of Semidefinite Relaxation MIMO Detectors for Higher-Order QAM,” 2008, submitted to IEEE Journal of Selected Topics in Signal Processing; available on arXiv.

[7] Z. Mao, X. Wang, and X. Wang, "Semidefinite Programming Relaxation Approach for Multiuser Detection of QAM Signals," IEEE Transactions on Wireless Communications, vol. 6, no. 12, pp. 4275-4279, 2007.

[8] A. Mobasher, M. Taherzadeh, R. Sotirov, and A. K. Khandani, "A Near-Maximum-Likelihood Decoding Algorithm for MIMO Systems Based on Semi-Definite Programming," IEEE Transactions on Information Theory, vol. 53, no. 11, pp. 38693886, 2007.

[9] M. Rudelson and R. Vershynin, "The Smallest Singular Value of a Random Rectangular Matrix," 2008, available on arXiv.

[10] N. D. Sidiropoulos and Z.-Q. Luo, "A Semidefinite Relaxation Approach to MIMO Detection for High-Order QAM Constellations," IEEE Signal Processing Letters, vol. 13, no. 9, pp. 525-528, 2006.

[11] A. M.-C. So, "Probabilistic Analysis of the Semidefinite Relaxation Detector in Digital Communications," 2008, manuscript.

[12] P. H. Tan and L. K. Rasmussen, "The Application of Semidefinite Programming for Detection in CDMA," IEEE Journal on Selected Areas in Communications, vol. 19, no. 8, pp. 14421449, 2001.

[13] D. Tse and P. Viswanath, Fundamentals of Wireless Communication. Cambridge University Press, 2005.

[14] L. Vandenberghe and S. Boyd, "Semidefinite Programming," SIAM Review, vol. 38, no. 1, pp. 49-95, 1996.

[15] S. Verdú, "Computational Complexity of Optimum Multiuser Detection," Algorithmica, vol. 4, pp. 303-312, 1989.

[16] — Multiuser Detection. Cambridge University Press, 1998.

[17] A. Wiesel, Y. C. Eldar, and S. Shamai (Shitz), "Semidefinite Relaxation for Detection of 16-QAM Signaling in MIMO Channels," IEEE Signal Processing Letters, vol. 12, no. 9, pp. 653-656, 2005.

[18] Y. Yang, C. Zhao, P. Zhou, and W. Xu, "MIMO Detection of 16-QAM Signaling Based on Semidefinite Relaxation," IEEE Signal Processing Letters, vol. 14, no. 11, pp. 797-800, 2007. 